\title{
Método para diseñar buenas prácticas de innovación educativa docente: percepción del profesorado
}

\author{
Method to design teaching educational innovation good practices: \\ teachers perception
}

\author{
Ángel Fidalgo Blanco ${ }^{1}$, María Luisa Sein-Echaluce Lacleta ${ }^{2}$, Francisco José García-Peñalvo ${ }^{3}$, \\ Ana María Balbín Bastidas ${ }^{4}$ \\ angel.fidalgo@upm.es,mlsein@unizar.es, fgarcia@usal.es, abalbin@pucp.edu.pe \\ ${ }^{1}$ Departamento de Ingeniería Geológica y Minera \\ Universidad Politécnica de Madrid \\ Madrid, España
${ }^{2}$ Departamento de Matemática Aplicada Universidad de Zaragoza
Zaragoza, España \\ ${ }^{3}$ Departamento de Informática y Automática \\ Universidad de Salamanca \\ Salamanca, España \\ ${ }^{4}$ Facultad de Gestión y Alta Dirección \\ Pontificia Universidad Católica del Perú \\ Lima, Perú
}

\begin{abstract}
Resumen- Gran parte de las universidades e instituciones educativas realizan convocatorias de innovación docente para fomentar que el profesorado la aplique en sus asignaturas. La realización de las propuestas de innovación educativa las realiza el profesorado, en algunos casos sin tener ni experiencia ni conocimientos en innovación educativa, pero con una gran voluntad por mejorar el aprendizaje en sus asignaturas. En este trabajo se presenta un método de aplicación de la innovación educativa que permite al profesorado seguir un conjunto de pasos y procedimientos para diseñar una propuesta de innovación educativa que sea eficaz, eficiente, transferible y sostenible.
\end{abstract}

Palabras clave: Innovación educativa, proyectos de innovación, método MAIN, indicadores de innovación

Abstract- A large part of universities and educational institutions make calls for teaching innovation to encourage teachers to apply it in their courses. The realization of the proposals of educational innovation is carried out by the teaching staff, in some cases without having neither experience nor knowledge in educational innovation, but with a great will to improve learning in their courses. This paper presents a method of application of educational innovation that allows teachers to follow a set of steps and procedures to design a proposal for educational innovation to be effective, efficient, transferable and sustainable.

Keywords: Educational innovation, innovation projects, MAIN method, indicators of innovation

\section{INTRODUCCIÓN}

El profesorado comenzó a realizar innovación educativa por iniciativa personal, motivado más por mejorar el aprendizaje de los estudiantes que por los posibles reconocimientos y méritos que suponía realizar la propia innovación (Fidalgo-Blanco, 2019a). La aplicación de la innovación educativa era iniciativa del profesorado y estudios sobre los modelos innovadores identificaban que el proceso se realizaba de abajo a arriba (Gautier Cruz et al., 2006); es decir, lo iniciaba el profesorado y el centro, en la mayoría de los casos, lo aceptaba.

Actualmente, la situación ha cambiado de forma considerable, la mayoría de las instituciones educativas (consejerías de educación, universidades y centros) apoyan y alientan a que el profesorado realice innovación educativa. Así mismo, cada vez son más las instituciones que realizan convocatorias públicas entre su profesorado; con ellas se pretende promover la innovación educativa, la mejora de la calidad docente y, en algunos casos, impulsar el plan estratégico de la institución educativa.

Además, en algunos países, como es el caso de España, las universidades suministran un soporte de innovación docente para su personal, ya que es su responsabilidad (ANECA, 2015). Cada vez son más las universidades que realizan convocatorias para promover la innovación educativa, así como jornadas para divulgar las innovaciones que se han realizado en las convocatorias y que las memorias de la innovación se publiquen en un repositorio institucional (Abadía et al., 2015).

Cuando la innovación educativa se aplica en el aula, se la suele denominar Innovación Docente o Innovación Educativa Docente (IED) (Fidalgo-Blanco, Sein-Echaluce, \& GarcíaPeñalvo, 2019) y esta tiene un conjunto de características que condicionan tanto su planificación como la aplicación y divulgación de las mismas.

Las características de la IED están ligadas a varios actores: al profesorado, a la institución y a la propia innovación. Es necesario conocerlas para poder realizar IED de forma eficiente y eficaz.

Una de las características asociadas al profesorado es la denominada línea de salida de la innovación. Esta consiste en 
la situación de partida, desde un momento determinado, de la que parten las organizaciones para desarrollar una determinada innovación que pueda asumir el sector al que va dirigida. Mientras que en las empresas que compiten por un mismo producto o servicio la línea de salida es muy similar para todas, en el sector educativo la línea de salida es muy distinta. Esto significa que, en el sector educativo, para hacer una misma innovación a parte del profesorado le costará mucho esfuerzo y a otra parte muy poco (Fidalgo-Blanco, 2019c). Dicho de otra forma, si se invierte un mismo esfuerzo en realizar innovación, el avance no será el mismo. Esto es así ya que la innovación la realiza el profesorado en su propia asignatura. Por tanto, la situación de la línea de salida depende de los conocimientos sobre innovación y sobre Tecnologías de la Información y la Comunicación (TIC) que posee el profesorado, del tipo de asignatura y del objetivo de innovación. La razón por la cual la línea de salida es semejante para, por ejemplo, las empresas que compiten en un sector y distinta para el profesorado es muy sencilla: el campo de acción de los primeros es global (el sector), mientras que para los segundos es local (la asignatura).

Una característica asociada a la institución educativa es que la convocatoria de innovación lleva asociados indicadores a los que el profesorado que hace una propuesta tendrá que adaptarse. El problema es que cada convocatoria institucional tiene sus propios indicadores y criterios, ya que las estrategias respecto a la calidad docente o innovación pueden ser distintas. Esto significa que lo que para una institución es considerada IED, para otra puede no serlo.

Una característica asociada a la propia innovación es el ciclo de la misma. Cualquier innovación parte de unos objetivos competitivos, se planifica y diseña, se desarrolla, se hace un prototipo, se valida $y$, si todo va bien, se pone en producción (García-Peñalvo, Sein-Echaluce, \& Fidalgo Blanco, 2015). El ciclo de una innovación está optimizado para que cuando la innovación salga al mercado, esta sea eficaz y eficiente. El ciclo también separa costes: no es lo mismo el coste asociado a la planificación, producción y validación, que el coste del producto final. En una IED el ciclo se reduce: por un lado, está la planificación y el diseño (propuesta de IED) y, por otra parte, se realiza de forma conjunta el desarrollo, el prototipo, la validación y la producción (dicho de otra forma, a la vez que se desarrolla, se aplica). El tener un ciclo de dos fases hace, por ejemplo, que sea muy difícil saber qué parte de la IED corresponde al desarrollo y qué parte a la aplicación. Esto causa que la mayoría del profesorado asocie un coste y esfuerzo excesivo para realizar una IED.

La innovación tecnológica, en cualquier sector industrial, tiene procedimientos estándares e indicadores comunes para su gestión (OECD, 2005). Dichos procedimientos e indicadores no son aplicables a la IED debido, entre otras cosas, a sus características específicas.

Uno de los escasos métodos creados para aplicar IED es el método MAIN (Método de Aplicación de la Innovación Educativa) (Fidalgo-Blanco, Sein-Echaluce Lacleta, \& GarcíaPeñalvo, 2018). Dicho método ha sido impartido en numerosos cursos y conferencias, y su validez ha sido contrastada a través de encuestas de percepción entre el profesorado universitario participante (Fidalgo-Blanco \& Sein-Echaluce, 2018a).

El objetivo de este artículo es aplicar el método MAIN para la planificación de proyectos IED para conseguir paliar el efecto negativo de las características expuestas. Los objetivos concretos son:

- Formular una propuesta de IED de forma global, independientemente de la asignatura, de los conocimientos del profesorado y de la problemática a resolver.

- Aportar evidencias en las propuestas de IED para que se pueda valorar la condición de buena práctica y de forma independiente a las distintas convocatorias institucionales.

- Aportar un método para que el profesorado pueda separar el esfuerzo de desarrollo y de aplicación de la IED.

Si se alcanzan estos objetivos se conseguirá que las innovaciones puedan tener un impacto global en el sector de la educación. Esto permitirá hacer planificaciones que puedan valorar cualquier institución, separar las fases de producción y aplicación (de esta forma, se puede saber el coste de desarrollo de la innovación y el coste de la aplicación) y, además, que sea independiente a la línea de salida de cada profesorado particular.

\section{CONTEXTO}

El modelo se aplica en un estudio de caso realizado a través de un curso de formación del profesorado de 40 horas de duración. En el mismo participaron 24 profesores y profesoras de la Universidad de la Coruña.

El curso se llevó a cabo de forma on-line y su estructura se basó en la realización, por parte de los asistentes, de actividades tanto grupales como individuales. Dichas actividades fueron revisadas y evaluadas $\mathrm{y}$, a partir de los resultados de las actividades, se realizaron puestas en común.

A partir de las puestas en común se elaboraron guías que reflejaron los distintos puntos que debía tener el resultado de la actividad. Las guías integran la experiencia colectiva de las personas que participaron en el curso (alumnado y profesorado). Dichas guías se pueden transferir y son las que representan el modelo del método y que se expone en la descripción a continuación.

\section{DESCRIPCIÓN}

Las fases del método MAIN se pueden considerar como una guía para que el profesorado pueda realizar una planificación de una buena práctica de IED. La primera parte comienza a partir de la necesidad concreta que tiene el profesorado en su asignatura y posteriormente se dan unas pautas para que, a partir de la necesidad identificada, se pueda establecer una planificación de una buena práctica de IED. A continuación, se describe cada una de las fases del método MAIN.

\section{A. Fase 1. Identificación del problema raíz}

Un problema raíz es una problemática presente en nuestras asignaturas originadas por el modelo educativo (FidalgoBlanco, 2019b). Esta problemática suele estar presente en cualquier asignatura independientemente, por ejemplo, de la calidad del profesorado. Al ser problemáticas comunes al modelo educativo, si el profesorado asocia su situación particular a esa problemática, la innovación pasa de ser una solución local a ser una solución global.

Al tratarse de una situación global, es más fácil conseguir la cooperación del profesorado para obtener una solución. La 
dificultad radica en formular esa solución en base a indicadores medibles y transferibles (aplicables en cualquier asignatura).

El resultado de esta fase es la generación de indicadores comunes y transferibles.

\section{B. Fase 2. Asociación del método de innovación más adecuado a la fase anterior}

En muchas ocasiones, se asocia la IED a utilizar las tecnologías más avanzadas o los métodos que son tendencia. Esto suele hacer que el profesorado se centre más en aspectos "vistosos" que en buscar el método que tenga una demostrada eficacia con los indicadores que le permitirán conocer el resultado de la innovación.

En esta fase se utiliza el conjunto de indicadores obtenidos en la fase anterior, como filtros para seleccionar el método o métodos que hayan demostrado su eficacia con los mismos.

\section{Fase 3. Ingeniería inversa de la tortilla de patata}

Para entender esta fase, se debe asociar una IED concreta a una tortilla de patata. A menudo el profesorado observa una experiencia de IED y se fija en los resultados de la misma (la tortilla de patata). Sin embargo, esto no suele aportar datos para conocer los pasos y esfuerzo para desarrollarla.

Por otro lado, si en lugar de observar la tortilla analizamos sus ingredientes (huevos, patatas, aceite, etc.) y las herramientas a utilizar (tenedor, sartén, plato, etc.) y, además, conocemos los procesos a realizar con esos ingredientes y herramientas (la receta), entonces podremos saber si seremos capaces de aplicar dicha innovación, de conocer el coste de los ingredientes, la complejidad de las tecnologías y el esfuerzo para realizar los procesos. Realmente se podría considerar como un ecosistema de aprendizaje, ya que intervienen distintos tipos de datos, tecnologías, procesos y usuarios.

La equivalencia en una IED respecto a los ingredientes, herramientas y receta de la tortilla se basa en lo siguiente:

- Los ingredientes. Son los tipos de contenidos que utiliza dicho método de IED y conocerlos permite comprender la posible transformación que se debe realizar en la asignatura en la que se desea innovar. Esto permite también conocer el coste de esa transformación.

- Las herramientas. Son las tecnologías y lo importante es conocer su funcionalidad. De esta forma se podrá identificar la tecnología por su funcionalidad y elegir la que más fácilmente se pueda utilizar, por ejemplo, una que se conozca o que ponga la institución a disposición del profesorado. Por tanto, no es necesario tener la última tecnología, sino la que más se adecúa a la función de la misma.

- La receta. Son las actividades que deben hacer el profesorado y el alumnado con los tipos de contenidos y las tecnologías. Las actividades suelen estar asociadas al método de innovación elegido $\mathrm{y}$, a menudo, es lo que distingue un método de otro.

D. Fase 4. Las estrategias

Hay al menos tres tipos de estrategias que se suelen asociar a una IED:

- Cambio de rol. Cualquier IED supone ciertos cambios tanto en el profesorado como en el alumnado. El éxito o fracaso de la innovación suele depender más del esfuerzo para asumir ese cambio de rol que del propio método de innovación docente (Fidalgo-Blanco, Sein-Echaluce, \& García-Peñalvo, 2017).

- Buena práctica. Hay indicadores que se asocian a una buena práctica de innovación docente: eficacia, eficiencia, sostenibilidad y transferibilidad (Fidalgo-Blanco \& SeinEchaluce Lacleta, 2018b). Así pues, el problema respecto a las propuestas de IED es cómo saber que esta presentará estos indicadores si aún no ha comenzado a desarrollarse. Por ejemplo, se define que para que una práctica se pueda considerar de innovación al menos debe durar tres años y para que sea relevante debe poder expandirse fuera de su ámbito (Infante \& Letelier, 2013)

- Divulgación científica. Uno de los indicadores externos más consolidados para conocer el éxito o fracaso de una IED es si esta se divulga en congresos internacionales o en revistas científicas indexadas. Tanto los congresos de calidad internacionales como las revistas indexadas tienen unos protocolos, filtros e indicadores para aceptar o rechazar la publicación. Por tanto, es necesario conocer los procesos científicos admitidos de forma internacional y asociarlos a la planificación de la propuesta.

La tabla 1 muestra las cuatro fases del método MAIN. En la primera columna se indica el número de fase, en la segunda columna las actividades principales de cada fase y, en la tercera columna, el objetivo de cada fase.

Tabla 1. Fases, actividades y objetivos del método MAIN

\begin{tabular}{|c|c|c|}
\hline & ACTIVIDADES & OBJETIVOS \\
\hline $\begin{array}{l}\mathbf{F} \\
\mathbf{A} \\
\mathbf{S}\end{array}$ & $\begin{array}{l}\text { Detección de } \\
\text { problemas raíz }\end{array}$ & $\begin{array}{l}\text { Definir el objetivo de la innovación, } \\
\text { aplicable de forma global (a todo el sector } \\
\text { de la educación). }\end{array}$ \\
\hline 1 & $\begin{array}{l}\text { Identificación de } \\
\text { indicadores de } \\
\text { mejoras }\end{array}$ & $\begin{array}{l}\text { Diseñar un mecanismo de medición de la } \\
\text { eficacia de la IED, facilitando su } \\
\text { transferencia. }\end{array}$ \\
\hline $\begin{array}{l}\mathbf{F} \\
\mathbf{A} \\
\mathbf{S} \\
\mathbf{E}\end{array}$ & $\begin{array}{l}\text { Asociación del } \\
\text { método de IED } \\
\text { más adecuado }\end{array}$ & $\begin{array}{l}\text { Identificar el método de innovación más } \\
\text { adecuado para alcanzar el objetivo de } \\
\text { innovación global en nuestra innovación } \\
\text { (y demostrarlo). } \\
\text { Optimizar el esfuerzo en el desarrollo de } \\
\text { la IED. }\end{array}$ \\
\hline $\begin{array}{l}\mathbf{F} \\
\mathbf{A} \\
\mathbf{S} \\
\mathbf{E}\end{array}$ & $\begin{array}{l}\text { Ingeniería } \\
\text { inversa de la } \\
\text { "tortilla } \\
\text { patata" }\end{array}$ & $\begin{array}{l}\text { Identificar el tipo de contenido con el qué } \\
\text { trabajar. } \\
\text { Identificar las TIC más adecuadas para } \\
\text { innovar. } \\
\text { Identificar los procesos de innovación } \\
\text { Separar las fases de desarrollo y } \\
\text { aplicación, valorando el esfuerzo y coste } \\
\text { de cada fase. } \\
\text { Generar una planificación de ciclo, a } \\
\text { través de un organigrama. }\end{array}$ \\
\hline $\begin{array}{l}\mathbf{F} \\
\mathbf{A} \\
\mathbf{S} \\
\mathbf{E}\end{array}$ & $\begin{array}{l}\text { Identificación de } \\
\text { indicadores de } \\
\text { buena práctica de } \\
\text { IED }\end{array}$ & $\begin{array}{l}\text { Demostrar la eficacia, eficiencia, } \\
\text { sostenibilidad y transferibilidad de la } \\
\text { innovación a realizar. }\end{array}$ \\
\hline 4 & $\begin{array}{l}\text { Identificación de } \\
\text { métodos de } \\
\text { divulgación } \\
\text { científica }\end{array}$ & $\begin{array}{l}\text { Diseñar la práctica de IED para que pueda } \\
\text { ser publicada en contextos científicos } \\
\text { acreditados (congresos internacionales y } \\
\text { revistas científicas). }\end{array}$ \\
\hline
\end{tabular}




\section{RESUltados}

En este estudio intervinieron 24 profesores universitarios pertenecientes a la Universidad de la Coruña que participaron en el curso a distancia sobre el método MAIN que se basa en una metodología participativa y activa. Cada fase tiene asociado un conjunto de actividades que generan unas evidencias con las que se trabaja de forma cooperativa e individual.

Fase 1. Primeramente, se definieron tres situaciones que suelen requerir IED para su mejora y que representan un problema raíz cada una de ellas. Las situaciones fueron:

- S1. Hábito pasivo del alumnado.

- S2. Seguimiento individual y en tiempo real para evaluar la competencia de trabajo en equipo, tanto de forma individual como grupal.

- S3. Adaptación del aprendizaje a las características del propio alumnado (aprendizaje personalizado).

Cada participante eligió de forma voluntaria el problema raíz con el que deseaba trabajar. La tabla 2 refleja el número de personas que asociaron su problemática a cada una de las situaciones.

Tabla 2. Número de personas que asociaron su problemática a la situación

\begin{tabular}{l|c}
\hline Situación & $\mathbf{N}^{\mathbf{0}}$ de personas \\
\hline S1 Hábito pasivo del alumnado & 14 \\
\hline $\begin{array}{l}\text { S2 Competencia trabajo en equipo } \\
\text { individual y grupal }\end{array}$ & 9 \\
\hline S3 Aprendizaje personalizado & 1 \\
\hline
\end{tabular}

A través de las actividades de trabajo se obtuvieron indicadores de mejora medibles que permiten calcular el impacto de la innovación en cada una de las situaciones. El conjunto de indicadores medibles se creó de forma cooperativa.

Fase 2. Identificar el método de IED más adecuado.

El objetivo de esta fase es identificar el método de IED más adecuado que permita trabajar con los indicadores obtenidos en la fase anterior. De esta forma se tienen garantías de que el método seleccionado es adecuado para cada situación individual del profesorado.

A través de las aportaciones realizadas por los participantes, se estableció un modelo aplicable a cualquier IED. Es decir, dicho modelo se puede utilizar para demostrar que se ha elegido el método de innovación más adecuado a cada necesidad concreta, independientemente del área de conocimiento, nivel académico y ámbito educativo.

La tabla 3 muestra un esquema de dicho modelo. El punto 1 de la tabla 3 se realiza durante la fase 1 del método MAIN. Se define el problema raíz con el que se va a trabajar y los indicadores de mejora que se van a utilizar para comprobar el impacto de la innovación educativa.

Los puntos 2 y 3 (tabla 3 ) se basan en la globalidad del problema raíz y los indicadores de mejora. Se debe buscar si todos se contemplan en planes institucionales o globales, para demostrar que la innovación propuesta no solamente es interesante para una asignatura determinada.
El punto 4 (tabla 3) busca aportar una descripción breve de la metodología de innovación elegida y relacionarla, al menos, con el problema raíz.

El punto 5 (tabla 3) busca demostrar que la metodología de innovación elegida es idónea y tiene garantías de que su aplicación tenga un impacto positivo. Para ello, se deben buscar referencias científicas que demuestren que:

- La metodología de innovación es percibida de forma positiva por el alumnado.

- Existe impacto en el aprendizaje (se debe relacionar con la actividad de la fase 1 donde se describe dicho impacto).

- Que mejora los indicadores seleccionados como "indicadores de mejora en la innovación propuesta".

El punto 6 (tabla 3) se utiliza en caso de que se elija un método de innovación concreto. Se debe describir en qué consiste el mismo.

El punto 7 (tabla 3) se basa en describir el contexto específico donde se va a aplicar la innovación (número de alumnos, tipo de asignatura, etc.).

Tabla 3. Esquema de realización de una propuesta de IED

1-Identificación del problema raíz y de los indicadores de mejora

2-Identificación (si procede) de otros contextos que tienen la misma problemática

3-Identificación del interés estratégico para la institución en la solución de la problemática detectada

4-Breve descripción del método de innovación elegido

5-Descripción genérica del método de IED (buscar referencias)

- 5-1 Percepción del alumnado

- 5-2 Impacto en el aprendizaje

- 5-3 Obtención de mejoras relacionadas con los indicadores de mejora

6- Especificación concreta del método (en caso de elegir un método de IED concreto)

7- Especificación del contexto donde se va a aplicar el método

Fase 3. Ingeniería inversa de la tortilla de patata

El objetivo de esta fase es que cada profesor asocie a la innovación elegida:

- El tipo de contenidos,

- Las tecnologías a utilizar y

- Las actividades que tendrá que realizar tanto el profesorado como el alumnado para aplicar la IED en su asignatura.

Respecto a las actividades, se identificaron cuatro tipos de actividad:

- A1. Actividades a realizar por el profesorado. Estas actividades se distribuyeron en: las que tiene que realizar el profesorado en el desarrollo y preparación de la innovación (Actividades A1.1), las realizadas durante la aplicación de la innovación (A1.2) y las realizadas para divulgar la innovación realizada (A1.3)

- A2. Actividades a realizar por el alumnado durante la aplicación de la innovación.

- A3. Actividades de medición. Son las actividades a realizar para medir el impacto de la IED. La medición del impacto 
se realiza a partir de los indicadores identificados en la fase 1.

Para cada una de las actividades identificadas se valoró el esfuerzo que el profesorado tendría que invertir en la realización de la misma.

La distribución de cada tipo de actividad a través de un cronograma se denomina cronograma de ciclo. La tabla 4 muestra un esquema general del diagrama de ciclo.

Tabla 4. Diagrama del ciclo de innovación

\begin{tabular}{l|l|l|l}
\hline Fase/meses & Desarrollo & Aplicación & Divulgación \\
\hline Tipo de & A1.1 & A1.2 & A1.3 \\
actividad & & A2 & \\
& & A3 & \\
\hline
\end{tabular}

En esta fase también se valora el esfuerzo para realizar cada una de las actividades y este puede ser de varios tipos:

- E1. Esfuerzo asociado a la novedad. Es un esfuerzo asociado a la dificultad del profesorado para manejar una tecnología. Puede ser muy alto (requerir mucho tiempo) si el profesorado nunca ha manejado una determinada tecnología y bajo si ya tiene experiencia en la misma. Este esfuerzo depende de cada profesorado.

- E2. Esfuerzo asociado al desarrollo de la actividad. Es el tiempo que cuesta realizar una determinada actividad en base a los procesos y requerimientos asociados a la misma. El esfuerzo es fijo, siempre que se realice esa actividad se requerirá el mismo esfuerzo.

- E3. Esfuerzo asociado a la medición y divulgación de la experiencia de la IED. Es un esfuerzo inicial que se requiere al aplicar por primera vez la innovación, y que en sucesivas aplicaciones no es necesario realizarlo.

El esfuerzo total que requiere realizar la IED es la suma de los esfuerzos descritos. Sin embargo, en caso de volver a aplicar la IED, el esfuerzo total se asocia concretamente al esfuerzo asociado al tipo E2.

Así pues, se identifican dos esfuerzos diferentes, el asociado al desarrollo, aplicación y divulgación de la IED y el esfuerzo asociado a replicar esa innovación en próximos cursos.

Fase 4. Estrategias

Esta fase tiene dos objetivos:

- Aportar indicadores que permitan valorar que la IED propuesta será una buena práctica.

- Definir un método de trabajo basado en el método científico.

Los indicadores de buena práctica se obtienen a partir de las siguientes evidencias.

- Eficacia: Se obtiene a partir de la medición de los indicadores de mejora identificados en la fase 1.

- Eficiencia: Identificando el esfuerzo para realizar la innovación por primera vez y el esfuerzo para replicarla.

- Sostenibilidad: A partir del esfuerzo temporal y del coste de las tecnologías a utilizar la próxima vez que se aplique la IED.
- Transferibilidad: A partir de la necesidad global del objetivo y los indicadores de mejora.

- Divulgación científica: Se obtiene a partir de las actividades de medición de cada indicador de mejora identificado y el método científico utilizado (estudio de caso, contraste de resultados y método cuasiexperimental).

\section{CONCLUSIONES}

Las IED están asociadas a cada asignatura y dependen de los conocimientos del profesorado que va a realizar dicha innovación. Esto ocasiona que dicha IED tenga un carácter local y sea muy complejo transferir sus resultados a otras asignaturas. Por tanto, es importante plantear las IED con alcance global y transferible. Los resultados de la fase 1 han demostrado que el objetivo de la innovación se puede plantear de forma común, así como los indicadores de mejora de forma independiente a la asignatura individual. Así pues, la aplicación del método MAIN en la planificación de una IED permite mejorar la asignatura de forma individual a la vez que avanzar en la mejora global de la educación. El trabajo cooperativo para definir los indicadores de mejora de forma global y la utilización de dichos indicadores para cada asignatura concreta así lo demuestran.

En algunas propuestas de IED se propone la utilización de tendencias de innovación (e incluso se mezclan varias). Estas propuestas se consideran innovación por la novedad de los métodos utilizados, pero no por la eficacia de los mismos para resolver una situación problemática de la asignatura. Los resultados de la fase 2 han demostrado que el profesorado puede elegir métodos de innovación en función de los indicadores de mejora, en lugar de la novedad del método. Esto fomenta la eficacia de la propuesta de IED realizada.

En las planificaciones de las IED se suele mezclar el desarrollo con la aplicación y divulgación de la misma. Esta situación hace que gran parte del profesorado no la lleve a cabo ya que la asocian con un alto coste y esfuerzo. Separar las fases de desarrollo, aplicación y divulgación permite conocer el coste y esfuerzo real para la realización de la IED. Los resultados de la fase 3 muestran que el profesorado ha conseguido identificar y separar esas tres fases. Así mismo, ha asociado, a cada una de las fases, actividades de distinto tipo asignando un esfuerzo concreto a cada una de ellas.

Se ha conseguido diseñar un modelo para la planificación de propuestas de IED de forma independiente a las especificaciones de la convocatoria. De hecho, el curso se ha desarrollado antes de que la Universidad de la Coruña haya realizado la convocatoria de proyectos de IED.

Para las instituciones y el profesorado es muy difícil demostrar que la propuesta de IED va a ser una buena práctica. Los resultados de la fase 4 han demostrado que en las propuestas se han aportado evidencias que demuestran o vislumbran la inclusión de indicadores de buena práctica. Por tanto, se han aportado evidencias de buena práctica antes de que finalice la misma.

Uno de los problemas principales para divulgar en contextos científicos las experiencias de IED es que estos exigen que se haya realizado un conjunto de actividades de medición, antes, durante y tras la finalización de la experiencia. Si no se ha realizado es muy difícil conseguir que se publique en contextos científicos. La aplicación del método MAIN para elaborar una 
propuesta de proyecto de innovación ha permitido incorporar dichas actividades de medición y, por consiguiente, aumentar la posibilidad de publicar la experiencia en contextos científicos.

\section{AgRAdECIMIENTOS}

Este trabajo ha sido parcialmente financiado por el Ministerio de economía y Competitividad de España, a través del proyecto DEFINES (Ref. TIN2016-80172-R) y al servicio de Innovación Educativa de la Universidad Politécnica de Madrid (Proyecto IE1819.0601 y Proyecto IE1819.0602). Los autores quieren agradecer el apoyo de los grupos de investigación GIDTIC (http://gidtic.com), GRIAL (http://grial.usal.es) y LITI (http://www.liti.es).

\section{REFERENCIAS}

Abadía, A.R., Beltrán, F., Bueno, C., Fidalgo Blanco, Á., Julián, J.A., Lerís, D., Lidón, I., R., \& R., Rivero, P., and Sein-Echaluce Lacleta, M. L. (2015). Repositorio de Buenas Prácticas de Innovación Docente de la Universidad de Zaragoza. (Fundación General de la Universidad Politécnica de Madrid, Ed.), Actas del III Congreso Internacional sobre Aprendizaje, Innovación y Competitividad. CINAIC 2015. Madrid: Á. Fidalgo Blanco, M.L. Sein-Echaluce Lacleta and F.J. GarcíaPeñalvo.

ANECA. (2015). Programa DOCENTIA.Programa de apoyo para la evaluación de la actividad docente del profesorado universitario.

http://www.aneca.es/Programas-deevaluacion/Evaluacioninstitucional/DOCENTIA/Documentacion-del-programa

Fidalgo-Blanco, Á. (2019a). Un método para conocer el estado del arte sobre la aplicación de la innovación educativa. La metáfora del camino. Zenodo https://doi.org/10.5281/ZENODO.2634583

Fidalgo-Blanco, Á. (2019b). Problemas "raíz" de la educación - Innovación Educativa. https://innovacioneducativa.wordpress.com/2018/06/19/ problemas-raiz-de-la-educacion/

Fidalgo-Blanco, Á. (2019c). ¿Por qué es tan distinta la innovación educativa a otros tipos de innovación? Innovación Educativa.

https://innovacioneducativa.wordpress.com/2019/07/10/ por-que-es-tan-distinta-la-innovacion-educativa-a-otrostipos-de-innovacion/

Fidalgo-Blanco, Á., \& Sein-Echaluce, M. L. (2018a). Método MAIN para planificar, aplicar y divulgar la innovación educativa. Education in the Knowledge Society (EKS),
19(2), 83-101.

https://doi.org/10.14201/eks201819283101

Fidalgo-Blanco, Á., \& Sein-Echaluce Lacleta, M. (2018b). ¿Qué hay que hacer para que una innovación educativa se consolide? In A. Fores Miravalles \& E. Subias Valeccillo (Eds.), Pedagogías emergentes: 14 preguntas para el debate (1st ed., p. 203). Barcelona: Ediciones Octaedro.

Fidalgo-Blanco, Á., Sein-Echaluce, M. L., \& García-Peñalvo, F. J. (2017). Inteligencia colectiva en el aula. Un paradigma cooperativo. In La innovación docente como misión del profesorado : CINAIC (pp. 1-5). Zaragoza: Servicio de Publicaciones Universidad. https://doi.org/10.26754/CINAIC.2017.000001_125

Fidalgo-Blanco, Á., Sein-Echaluce Lacleta, M. L., \& GarcíaPeñalvo, F. J. (2018). Method for Applying Innovation in educatioN (MAIN). Salamanca. https://doi.org/10.5281/zenodo.1439134

Fidalgo-Blanco, Á., Sein-Echaluce, M. L., \& García-Peñalvo, F. . (2019). ¿Pueden las tendencias de innovación educativa predecir los cambios que transformarán el modelo educativo?? https://doi.org/10.5281/ZENODO.2672967

García-Peñalvo, F. J. (2017). The Future of Institutional Repositories. Education in the Knowledge Society (EKS), 18(4), 7-19. https://doi.org/10.14201/eks2017184719

García-Peñalvo, F. J., Sein-Echaluce, M. L., \& Fidalgo Blanco, Á. (2015). Educational Innovation Management. A Case Study at the University of Salamanca. In G.R. Alves and M.C. Felgueiras (Ed.), Proceedings of the Third International Conference on Technological Ecosystems for Enhancing Multiculturality (TEEM'15) (pp. 151-158). New York: ACM.

Gautier Cruz, E., León Cháves, E., Gómez Puente, S. M., Jamil Cury, C. R., Merodo, A., Torrecilla Murillo, F. J., Pilar Unda Bernal, M. del. (2006). Modelos innovadores en la formación inicial docente. (F. J. C. Murillo Torrecilla, Ed.). UNESCO, Oficina Regional de Educación para América Latina y el Caribe.

Infante, M. I., \& Letelier, M. E. (2013). AlfAbetizAción y educAción. Lecciones aprendidas desde la práctica innovadora en América Latina y el Caribe. Santiago, Chile: UNESCO.

OECD. (2005). Oslo Manual. Guidelines for Collecting and Interpreting Innovation (3rd ed.). OECD Publishing. https://doi.org/10.1787/9789264013100-en 